\title{
Theories of the initial mass function
}

\author{
Patrick Hennebelle ${ }^{1}$ and Gilles Chabrier ${ }^{2}$ \\ ${ }^{1}$ Laboratoire de radioastronomie, Ecole normale supérieure and Observatoire de Paris, \\ UMR CNRS 811224 rue Lhomond, 75231 Paris Cedex 05, France \\ email: patrick.hennebelle@ens.fr \\ ${ }^{2}$ CRAL, Ecole normale supérieure de Lyon, UMR CNRS 5574 \\ Univesrité de Lyon, 69364 Lyon Cedex 07, France \\ email: chabrier@ens-lyon.fr
}

\begin{abstract}
We review the various theories which have been proposed along the years to explain the origin of the stellar initial mass function. We pay particular attention to four models, namely the competitive accretion and the theories based respectively on stopped accretion, MHD shocks and turbulent dispersion. In each case, we derive the main assumptions and calculations that support each theory and stress their respective successes and failures or difficulties.
\end{abstract}

Keywords. STARS: formation, ISM: clouds, gravitation, turbulence

\section{Introduction}

Stars are the building blocks of our universe and understanding their formation and evolution is one of the most important problems of astrophysics. Of particular importance is the problem of the initial mass function of stars (Salpeter 1955, Scalo 1986, Kroupa 2002, Chabrier 2003) largely because the star properties, evolution and influence on the surrounding interstellar medium strongly depend on their masses. It is generally found that the number of stars per logarithmic bin of masses, $d N / d \log M$, can be described by a lognormal distribution below $1 M_{\odot}$, peaking at about $\simeq 0.3 M_{\odot}$, and a power-law of slope -1.3 for masses between 1 and $10 M_{\odot} \dagger$ (e.g. Chabrier 2003). It should be stressed that the IMF of more massive stars is extremely poorly known.

Several theories have been proposed to explain the origin of the IMF, invoking various physical processes that we tentatively classify in four categories: theories based on recursive fragmentation or pure gravity (e.g. Larson 1973), theories based on pure statistical argument, invoking the central limit theorem (e.g. Zinnecker 1984, Elmegreen 1997, Adams \& Fatuzzo 1996), theories based on accretion and, finally, theories invoking the initial Jeans mass in a fluctuating environment. We will focus on the two latter ones, which appear to be favored in the modern context of star formation.

\section{Theories based on accretion}

\subsection{Competitive accretion}

The theory of competitive accretion has been originally proposed by Zinnecker (1982) and Bonnell et al. (2001). It has then been used to interpret the series of numerical simulations similar to the ones performed by Bate et al. (2003).

$\dagger$ It should be kept in mind that the initial mass function is measured only up to about 8 $M_{\odot}$, in young stellar clusters, and is inferred only indirectly for larger masses (see e.g. Kroupa 2002) 
The underlying main idea is that the accretion onto the star is directly linked to its mass in such a way that massive stars tend to accrete more efficiently and thus become disproportionally more massive than the low mass stars. The accretion rate is written as:

$$
\dot{M}_{*}=\pi \rho V_{r e l} R_{a c c}^{2}
$$

where $\rho$ is the gas density, $V_{\text {rel }}$ is the relative velocity between the star and the gas while $R_{a c c}$ is the accretion radius. Bonnell et al. (2001) consider two situations, namely the cases where the gravitational potential is dominated by the gas or by the stars.

\subsubsection{Gas dominated potential}

Let $R$ be the spherical radius, $\rho$ the gas density and $n_{*}$ the number density of stars. In the gas dominated potential case, Bonnell et al. (2001) assume, following Shu (1977), that the gas density profile is proportional to $R^{-2}$. They further assume that $n_{*} \propto R^{-2}$. The accretion radius, $R_{a c c}$ is assumed to be equal to the tidal radius given by

$$
R_{\text {tidal }} \simeq 0.5\left(\frac{M_{*}}{M_{\text {enc }}}\right)^{1 / 3} R,
$$

where $M_{e n c}$ is the mass enclosed within radius $R$. This choice is motivated by the fact that a fluid particle located at a distance from a star smaller than $R_{\text {tidal }}$ is more sensitive to the star than to the cluster potential and will thus be accreted onto this star.

The mass of gas within a radius $R, M(R)$, is proportional to $R$ (since $\rho \propto R^{-2}$ ). The infall speed is about $V_{i n} \simeq \sqrt{G M(R) / R}$, and, assuming that the stars are virialized, one gets $V_{\text {rel }} \simeq V_{\text {in }}$. The number of stars, $d N_{*}$, located between $R$ and $R+d R$ is given by the relation $d N_{*}=n_{*}(R) \times 4 \pi R^{2} d R \propto d R$. Thus eqn 2.1 combined with the expression of $V_{\text {rel }}$ and $R_{a c c}$ leads to the relation $\dot{M}_{*} \propto\left(M_{*} / R\right)^{2 / 3}$ and, after integration, $M_{*} \propto R^{-2}$, $R \propto M_{*}^{-1 / 2}$. Consequently, we obtain:

$$
d N \propto M_{*}^{-3 / 2} d M_{*}
$$

Even though the mass spectrum is too shallow compared to the fiducial IMF, $d N / d M \propto$ $M^{-2.3}$, it is interesting to see that this power law behaviour can be obtained from such a simple model. Note, however, that the model implies that stars of a given mass are all located in the same radius, which points to a difficulty of the model.

\subsubsection{Star dominated potential}

When the potential is dominated by the stars located in the centre of the cloud, the density is given by $\rho \propto R^{-3 / 2}$, which corresponds to the expected density distribution after the rarefaction wave has propagated away (Shu 1977). The velocity is still assumed to be $V_{\text {rel }} \propto R^{-1 / 2}$. The accretion radius is now supposed to correspond to the BondiHoyle radius as the gas and the star velocities are no longer correlated. This leads to $\dot{M}_{*}=\pi \rho V_{\text {rel }} R_{B H}^{2}$ where $R_{B H} \propto M_{*} / V_{\text {rel }}^{2}$. It follows

$$
\dot{M}_{*} \propto M_{*}^{-2} \text {. }
$$

One can then show (Zinnecker 1982, Bonnell et al. 2001) that under reasonable assumptions, $d N \propto M_{*}^{2} d M_{*}$. This estimate is in better agreement with the Salpeter exponent, although still slightly too shallow. These trends seem to be confirmed by the simulations performed by Bonnell et al. (2001) which consists in distributing 100 sink particles in a cloud of total mass about 10 times the total mass of the sinks initially (their Fig. 3). 


\subsubsection{Difficulties of the competitive accretion scenario}

As obvious from the previous analytical derivations, finding an explanation for the Salpeter exponent with the competitive accretion scenario appears to be difficult, even though numerical simulations (as the ones presented in Bonnell et al. 2001) seem to successfully achieve this task. However, although the IMF exponent is close to the Salpeter one in the star dominated potential case, this scenario entails by construction the BondiHoyle accretion, which is at least a factor 3 lower than the mass infall rate resulting from gravitational collapse at the class $\mathrm{O}$ and I stages and leads to too long accretion times compared with observations (André et al. 2007, 2009). Another difficulty of this scenario is that it does not explain the peak of the IMF which might be related to the Jeans mass (see $\S 3$ ). Finally, it is not clear that competitive accretion can work in the case of non-clustered star formation, for which the gas density is much too small. As no evidence for substantial IMF variation among different regions has yet been reported, this constitutes a difficulty for this model as a general model for star formation. Perhaps this scenario applies well to the formation of massive stars.

\subsection{Stopped accretion}

The principle of this type of models is to assume that the accretion of gas onto the stars or the dense cores is a non-steady process, stopped because of either the finite reservoir of mass or the influence of an outflow which sweeps up the remaining gas within the vicinity of the accreting protostar.

The first studies were performed by Silk (1995) and Adams \& Fatuzzo (1996). They first relate the mass of the stars to the physical parameters of the cloud such as sound speed and rotation and then assume that an outflow whose properties are related to the accretion luminosity stops the cloud collapse. Using the Larson (1981) relations, they can link all these parameters to the clump masses. Since the mass spectrum of these latter is known (e.g. Heithausen et al. 1998), they infer the IMF.

A statistical approach has been carried out by Basu \& Jones (2004). These authors assume that the dense core distribution is initially lognormal, justifying it by the large number of processes that control their formation (and invoking the central limit theorem). Then, they argue that the cores grow by accretion and postulate that the accretion rate is simply proportional to their mass, $\dot{M}=\gamma M \rightarrow M(t)=M_{0} \exp (\gamma t)$, leading to $\log M=\mu=\mu_{0}+\gamma t$. Finally, they assume that accretion is lasting over a finite period of time given by $f(t)=\delta \exp (-\delta t)$. The star mass distribution is thus obtained by summing over the accretion time distribution.

$$
\begin{aligned}
f(M) & =\int_{0}^{\infty} \frac{\delta \exp (-\delta t)}{\sqrt{2 \pi} \sigma_{0} M} \exp \left(-\frac{\left(\ln M-\mu_{0}-\gamma t\right)^{2}}{2 \sigma_{0}^{2}}\right) d t \\
& =\frac{\alpha}{2} \exp \left(\alpha \mu_{0}+\alpha^{2} \sigma_{0}^{2} / 2\right) M^{-1-\alpha} \operatorname{erf}\left(\frac{1}{\sqrt{2}}\left(\alpha \sigma_{0}-\frac{\ln M-\mu_{0}}{\sigma_{0}}\right)\right)
\end{aligned}
$$

where $\alpha=\delta / \gamma$ and $\sigma_{0}$ characterizes the width of the initial dense core distribution. As $\delta$ and $\alpha$ are controlled by the same types of processes, their ratio is expected to be of the order of unity and thus $f(M)$ exhibits a powerlaw behaviour close to the fiducial IMF.

In a recent study, Myers (2009) develops similar ideas in more details, taking into account the accretion coming from the surrounding background. Adjusting two parameters, he reproduces quite nicely the observed IMF (his figure 5).

A related model has also been developed by Bate \& Bonnell (2005) based on an idea proposed by Price \& Podsiadlowski (1995). They consider objects that form by fragmentation within a small cluster and are ejected by gravitational interaction with the other 
fragments, which stops the accretion process. Assuming a lognormal accretion rate and an exponential probability of being ejected, these authors construct a mass distribution that can fit the IMF for some choices of parameters.

In summary, the stopped accretion scenario presents interesting ideas and, providing (typically 2 or more) adequate adjustable parameters, can reproduce reasonably well the IMF. However, the very presence of such parameters, which characterizes our inability to precisely determine the processes that halt accretion, illustrates the obvious difficulties of this class of models, and their lack of predictive power and accuracy.

\section{Gravo-turbulent theories}

While in the accretion models, turbulence is not determinant, it is one of the essential physical processes for the two theories presented in this section, although the role it plays differs in both models, as shown below. The theories proposed along this line seemingly identify cores or pre-cores and are motivated by the strong similarity between the observed CMF and the IMF (e.g. André et al. 2010).

The first theory which combined turbulence and gravity was proposed by Padoan et al. (1997). In this paper, the authors consider a lognormal density distribution - density PDF computed from numerical simulations (e.g. Vázquez-Semadeni 1994, Kritsuk et al. 2007, Schmidt et al. 2009, Federrath et al. 2010) are indeed nearly lognormal - and select the regions of the flow which are Jeans unstable. By doing so, they get too stiff an IMF (typically $d N / d M \propto M^{-3}$ ) but nevertheless find a lognormal behaviour at small masses, a direct consequence of the lognormal density distribution, and a powerlaw one at large masses.

\subsection{Formation of cores by MHD shocks}

The idea developed by Padoan \& Nordlund (2002) is slightly different. These authors consider a compressed layer formed by ram pressure in a weakly magnetized medium. They assume that the magnetic field is parallel to the layer and thus perpendicular to the incoming velocity field. The postshock density, $\rho_{1}$, the thickness of the layer, $\lambda$, and the postshock magnetic field, $B_{1}$, can be related to the Alfvénic Mach numbers, $\mathcal{M}_{a}=v / v_{a}$ ( $v$ is the velocity and $v_{a}$ the Alfvén speed), and preshocked quantities, $\rho_{0}$ and $B_{0}$ according to the shock conditions:

$$
\rho_{1} / \rho_{0} \simeq \mathcal{M}_{a}, \lambda / L \simeq \mathcal{M}_{a}^{-1}, B_{1} / B_{0} \simeq \mathcal{M}_{a},
$$

where $L$ is the scale of the turbulent fluctuation. Note that for classical hydrodynamical isothermal shocks, the jump condition is typically $\propto \mathcal{M}^{2}$. The dependence on $\mathcal{M}_{a}$ instead of $\mathcal{M}^{2}$ stems from the magnetic pressure which is quadratic in $B$. As we will see, this is a central assumption of this model.

The typical mass of this perturbation is expected to be

$$
m \simeq \rho_{1} \lambda^{3} \simeq \rho_{0} \mathcal{M}_{a}\left(\frac{L}{\mathcal{M}_{a}}\right)^{3} \simeq \rho_{0} L^{3} \mathcal{M}_{a}^{-2} .
$$

As the flow is turbulent, the velocity distribution depends on the scale and $v \simeq L^{\alpha}$, with $\alpha=(n-3) / 2, E(k) \propto k^{-n}$ being the velocity powerspectrum $\dagger$. Combining these expressions with eqn (3.2), they infer

$$
m \simeq \frac{\rho_{0} L_{0}^{3}}{\mathcal{M}_{a, 0}}\left(\frac{L}{L_{0}}\right)^{6-n},
$$

$\dagger n$ is denoted $\beta$ in Padoan \& Nordlund (2002), more precisely $n-2=\beta$ 
where $L_{0}$ is the largest or integral scale of the system and $\mathcal{M}_{a, 0}$ the corresponding Mach number. To get a mass spectrum, it is further assumed that the number of cores, $N(L)$, formed by a velocity fluctuation of scale $L$, is proportional to $L^{-3}$. Combining this last relation with eqn (3.3) leads to

$$
N(m) d \log m \simeq m^{-3 /(6-n)} d \log m .
$$

For a value of $n=3.74$ (close to what is inferred from 3D numerical simulations), one gets $N(m) \simeq m^{-1.33}$, very close to the Salpeter exponent.

So far, gravity has not been playing any role in this derivation and the mass spectrum that is inferred is valid for arbitrarily small masses. In a second step, these authors consider a distribution of Jeans masses within the clumps induced by turbulence. As the density in turbulent flows presents a lognormal distribution, they assume that this implies a lognormal distribution of Jeans lengths and they multiply the mass spectrum (3.4) by a distribution of Jeans masses, which leads to

$$
N(m) d \log m \simeq m^{-3 /(6-n)}\left(\int_{0}^{m} p\left(m_{J}\right) d m_{J}\right) d \log m .
$$

The shape of the mass spectrum stated by eqn 3.5 is very similar to the observed IMF (see for example the figure 1 of Padoan \& Nordlund 2002).

Note, however, that difficulties with this theory have been pointed out by McKee \& Ostriker (2007) and Hennebelle \& Chabrier (2008). Eqn (3.1), in particular, implies that in the densest regions where dense cores form, the magnetic field is proportional to the density, in strong contrast with what is observed both in simulations (Padoan \& Nordlund 1999, Hennebelle et al. 2008) and in observations (e.g. Troland \& Heiles 1986). This is a consequence of the assumption that the magnetic field and the velocity field are perpendicular, which again is not the trend observed in numerical simulations. In both cases, it is found that at densities lower than about $10^{3} \mathrm{~cm}^{-3}, B$ depends only weakly on $n$ while at higher densities, $B \propto \sqrt{\rho}$. This constitutes a problem for this theory, as the index of the power law slope is a direct consequence of eqn (3.1). Assuming a different relation between $B$ and $\rho$, as the aforementioned observed one, would lead to a slope stiffer than the Salpeter value. Furthermore, the Salpeter IMF is recovered in various purely hydrodynamical simulations (e.g. Bate et al. 2003), while the Padoan \& Nordlund theory predicts a stiffer distribution $\left(d N / d M \propto M^{-3}\right)$ in the hydrodynamical case. Another important shortcoming of this theory is that it predicts that turbulence, by producing overdense, gravitationally unstable areas, always promotes star formation, while it is well established from numerical simulations that the net effect of turbulence is to reduce the star formation efficiency (e.g. MacLow \& Klessen 2004).

\subsection{Turbulent dispersion}

Recently, Hennebelle \& Chabrier (2008, 2009, HC08, HC09) proposed a different theory which consists in counting the mass of the fluid regions within which gravity dominates over the sum of all supports, thermal, turbulent and magnetic, according to the Virial condition. In this approach, the role of turbulence is dual: on one hand it promotes star formation by locally compressing the gas but on the other hand, it also quenches star formation because of the turbulent dispersion of the flow, which is taken into account in the selection of the pieces of fluid that collapse.

The theory is formulated by deriving an extension of the Press \& Schechter (1974, PS) statistical formalism, developed in cosmology. The two major differences are (i) the underlying density field, characterized by small and Gaussian fluctuations in the cosmological case while lognormal in the star formation case, and (ii) the selection criterion, 
a simple scale-free density threshold in cosmology while scale-dependent, based on the Virial theorem in the second case. That is, fluid particles which satisfy the criterion (see $\mathrm{HC} 08$ )

$$
\left\langle V_{\mathrm{rms}}^{2}\right\rangle+3\left(C_{s}^{e f f}\right)^{2}<-E_{\mathrm{pot}} / M
$$

are assumed to collapse and form a prestellar bound core. The turbulent rms velocity obeys a power-law correlation with the size of the region, the observed so-called Larson relation, $\left\langle V_{\mathrm{rms}}^{2}\right\rangle=V_{0}^{2} \times\left(\frac{R}{1 \mathrm{pc}}\right)^{2 \eta}$, with $V_{0} \simeq 1 \mathrm{~km} \mathrm{~s}^{-1}$ and $\eta \simeq 0.4-0.5$ (Larson 1981).

The principle of the method is the following. First, the density field is smoothed at a scale $R$, using a window function. Then, the total mass contained in areas which, at scale $R$, have a density contrast larger than the specified density criterion $\delta_{R}^{c}$, is obtained by integrating accordingly the density PDF. This mass, on the other hand, is also equal to the total mass located in structures of mass larger than a scale dependent critical mass $M_{R}^{c}$, which will end up forming structures of mass smaller than or equal to this critical mass for collapse (see $\mathrm{HC} 08 \S 5.1$ ).

$$
\int_{\delta_{R}^{c}}^{\infty} \bar{\rho} \exp (\delta) \mathcal{P}_{R}(\delta) d \delta=\int_{0}^{M_{R}^{c}} M^{\prime} \mathcal{N}\left(M^{\prime}\right) P\left(R, M^{\prime}\right) d M^{\prime} .
$$

In this expression, $M_{R}^{c}$ is the mass which at scale $R$ is gravitationally unstable, $\delta_{R}^{c}=$ $\log \left(\rho_{R}^{c} / \bar{\rho}\right)$ and $\rho_{R}^{c}=M_{R}^{c} /\left(C_{m} R^{3}\right), C_{m}$ being a dimensionless coefficient of order unity. $\mathcal{P}_{R}$ is the (turbulent) density PDF, assumed to be lognormal, while $P\left(R, M^{\prime}\right)$ is the conditional probability to find a gravitationally unstable mass, $M^{\prime}$ embedded into $M_{R}^{c}$ at scale $R$, assumed to be equal to 1 (see HC08 $§ 5.1 .2$ and App. D). Note that this expression is explicitly solving the cloud in cloud problem as the mass which is unstable at scale $R$ is spread over the structures of masses smaller than $M_{R}^{c}$ (right hand side integral). Therefore, by construction, all the gravitationally unstable regions in the parent clump that will eventually collapse to form individual prestellar cores are properly accounted for in this theory (see $\mathrm{HC} 08 \S 5.1$ ).

Taking the derivative of eqn (3.7) with respect to $R$, we obtain the mass spectrum

$$
\mathcal{N}\left(M_{R}^{c}\right)=\frac{\bar{\rho}}{M_{R}^{c}} \frac{d R}{d M_{R}^{c}}\left(-\frac{d \delta_{R}^{c}}{d R} \exp \left(\delta_{R}^{c}\right) \mathcal{P}_{R}\left(\delta_{R}^{c}\right)+\int_{\delta_{R}^{c}}^{\infty} \exp (\delta) \frac{d \mathcal{P}_{R}}{d R} d \delta\right) .
$$

While the second term is important to explain the mass spectrum of unbound clumps defined by a uniform density threshold (as the CO clumps), it plays a minor role for (Virial defined) bound cores and can generally be dropped (see HC08 for details).

After soma algebra and proper normalisation, one gets

$$
\mathcal{N}(\widetilde{M})=2 \mathcal{N}_{0} \frac{1}{\widetilde{R}^{6}} \frac{1+(1-\eta) \mathcal{M}_{*}^{2} \widetilde{R}^{2 \eta}}{\left[1+(2 \eta+1) \mathcal{M}_{*}^{2} \widetilde{R}^{2 \eta}\right]} \times\left(\frac{\widetilde{M}}{\widetilde{R}^{3}}\right)^{-\frac{3}{2}-\frac{1}{2 \sigma^{2}} \ln \left(\widetilde{M} / \widetilde{R}^{3}\right)} \times \frac{\exp \left(-\sigma^{2} / 8,3,9\right)}{\sqrt{2 \pi} \sigma}
$$

where $\widetilde{R}=R / \lambda_{J}^{0}, \delta_{R}^{c}=\ln \left\{\left(1+\mathcal{M}_{*}^{2} \widetilde{R}^{2 \eta}\right) / \widetilde{R}^{2}\right\}, \mathcal{N}_{0}=\bar{\rho} / M_{J}^{0}$ and $M_{J}^{0}, \lambda_{J}^{0}$ denote the usual thermal Jeans mass and Jeans length, respectively, and

$$
\widetilde{M}(R)=M / M_{J}^{0}=\widetilde{R}\left(1+\mathcal{M}_{*}^{2} \widetilde{R}^{2 \eta}\right)
$$

denotes the unstable mass at scale $R$ in the turbulent medium. The theory is controlled 
by two Mach numbers, namely

$$
\mathcal{M}_{*}=\frac{1}{\sqrt{3}} \frac{V_{0}}{C_{s}}\left(\frac{\lambda_{J}^{0}}{1 \mathrm{pc}}\right)^{\eta} \approx(0.8-1.0)\left(\frac{\lambda_{J}^{0}}{0.1 \mathrm{pc}}\right)^{\eta}\left(\frac{C_{s}}{0.2 \mathrm{~km} \mathrm{~s}^{-1}}\right)^{-1}
$$

defined as the non-thermal velocity to sound speed ratio at the mean Jeans scale $\lambda_{J}^{0}$ (and not at the local Jeans length), and the usual Mach number, $\mathcal{M}$, which represents the same quantity at the scale of the turbulence injection scale, $L_{i}$, assumed to be the characteristic size of the system, $\mathcal{M}=\frac{\left\langle V^{2}\right\rangle^{1 / 2}}{C_{s}}$.

The global Mach number, $\mathcal{M}$, broadens the density PDF, as $\sigma^{2}=\ln \left(1+b^{2} \mathcal{M}^{2}\right)$, illustrating the trend of supersonic turbulence to promote star formation by creating new overdense collapsing seeds.

The effect described by $\mathcal{M}_{*}$ is the additional non thermal support induced by the turbulent dispersion. In particular, at large scales the net effect of turbulence is to stabilize pieces of fluid that would be gravitationally unstable if only the thermal support was considered. This is illustrated by eqn (3.10) which reduces to $\widetilde{M}=\widetilde{R}$ when $\mathcal{M}_{*}=0$. In particular, for a finite cloud size, the gas whose associated turbulent Jeans length is larger than the cloud size is not going to collapse.

When $\mathcal{M}_{*} \ll 1$, i.e. the turbulent support is small compared to the thermal one, eqn (3.9) shows that the CMF at large masses is identical to the Padoan et al. (1997) result, i.e. $d N / d \log M \propto M^{-2}$. On the other hand, when $\mathcal{M}_{*} \simeq 1, d N / d \log M \propto$ $M^{-(n+1) /(2 n-4)}$, where the index of the velocity powerspectrum $n$ is related to $\eta$ by the relation $\eta=(n-3) / 2$ (see HC08). As $n \simeq 3.8-3.9$ in supersonic turbulence simulations (e.g. Kritsuk et al. 2007), turbulent dispersion leads to the correct Salpeter slope and Larson velocity-size relation.

Comparisons with the Chabrier (2003) IMF have been performed for a series of cloud parameters (density, size, velocity dispersion) and good agreement has been found (HC09) for clouds typically 3 to 5 times denser than the mean density inferred from Larson (1981) density-size relation. Comparisons with numerical simulations have also been performed. In particular, Schmidt et al. (2010), performing supersonic isothermal simulations with various forcing, have computed the mass spectrum of cores supported either by pure thermal support or by turbulent plus thermal support. Their converged simulations show very good quantitative agreement with the present theory, confirming that turbulent support is needed to yield the Salpeter index. Note that Schmidt et al. (2010) use for the density PDF the one they measure in their simulations which is nearly, but not exactly lognormal. Comparisons with the results of SPH simulations (Jappsen et al. 2005) including self-gravity and thermal properties of the gas have also been found to be quite successful (HC09).

\subsection{Difficulties of the gravo-turbulent theories}

One natural question about any IMF theory is to which extent it varies with physical conditions. Indeed, there is strong observational support for a nearly invariant form and peak location of the IMF in various environments under Milky Way like conditions (see e.g. Bastian et al. 2010). Jeans length based theories could have difficulty with the universality of the peak position, since it is linked to the Jeans mass which varies with the gas density. Various propositions have been made to alleviate this problem. Elmegreen et al. (2008) and Bate (2009) propose that the gas temperature may indeed increase with density, resulting in a Jeans mass which weakly depends on the density, while HC08 propose that for clumps following Larson relations, there is a compensation between the 
density dependence of the Jeans mass and the Mach number dependence of the density $\mathrm{PDF}$, resulting in a peak position that is insensitive to the clump size.

A related problem is the fact that massive stars are often observed to be located in the densest regions, where the Jeans mass is smaller. Indeed, $M_{J} \propto \rho^{-1 / 2}$ when a purely thermal support is considered, whereas $M_{J} \propto \rho^{-2}$ when turbulence is taken into account (assuming that $V \propto L^{0.5}$ ) (see HC09). This constitutes a difficulty for theories based on Jeans mass although, as seen above, the issue is much less severe when turbulent support is considered as massive stars can be formed at densities only few times smaller than the densities at which low mass stars form. Another possibility is that dynamical interactions between young protostars may lead to the migration of massive stars in the center of the gravitational well.

Furthermore, the dependence of the freefall time on the Jeans mass should also modify the link between the CMF and the IMF, as pointed out by Clark et al. (2007). This is particularly true for theories which invoke only thermal support. When turbulent support is included, the free-fall time is found to depend only weakly on the mass, with $t_{f f} \propto M^{1 / 4}$ (see McKee \& Tan 2003 and HC09 App. C), resolving this collapsing time problem. We stress that this time represents the time needed for the whole turbulent Jeans mass to be accreted. It is certainly true that, within this turbulent Jeans mass, small structures induced by turbulent compression will form rapidly. Their total mass, however, is expected to represent only a fraction of the total turbulent Jeans mass because the net effect of turbulence is to decrease the star formation efficiency (see HC08).

Generally speaking, the fragmentation that occurs during the collapse could constitute a problem for theories invoking Jeans masses. Although this problem is far from being settled, it should be stressed that such a fragmentation process is not incompatible with the calculations performed by $\mathrm{HC} 08$. As shown by eqn 3.7 , the presence of small self-gravitational condensations induced by turbulence at the early stages of star formation and embedded into larger ones is self-consistently taken into account in the theory. Moreover, the SPH simulations performed by Smith et al. (2008) show a clear correlation between the initial masses within the gravitational well and the final sink masses up to a few local freefall times (see Chabrier \& Hennebelle 2010 for a quantitative analysis), suggesting that the initial prestellar cores do not fragment into many objects. As time goes on, the correlation becomes weaker but seems to persist up to the end of their run. Massive stars, on the other hand, are weakly correlated with the mass of the potential well in which they form. Whether their mass was contained into a larger more massive well with which the final sink mass would be well correlated remains an open issue, which needs to be further investigated. At last, both the magnetic field (e.g. Machida et al. 2005, Hennebelle \& Teyssier 2008) and the radiative feedback (Bate 2009, Offner et al. 2009) will reduce the fragmentation, suggesting that the core-sink correlation found in Smith et al. (2008) should improve if such processes were included. Clearly, these questions require careful investigations.

\section{Conclusion}

We have reviewed the most recent theories which have been proposed to explain the origin of the IMF. Due to limited space, it was not possible to cover all of them (e.g. Kunz \& Mouschovias 2009). Two main categories received particular attention: the theories based on accretion and the ones based explicitly on turbulence. It should be stressed that these theories are not all exclusive from each other and may apply in different ranges of mass. For instance, the turbulent dispersion theory calculates the distribution of the initial mass accretion reservoirs; it is not incompatible with the stopped accretion theories 
and with the competitive accretion as long as mass redistribution/competition occurs within one parent core reservoir. The question as to whether one of these mechanisms is dominant is yet unsettled. Detailed comparisons between systematic sets of simulations, as done in Schmidt et al. (2010), or observations, and the various analytical predictions is clearly mandatory to make further progress.

\section{References}

Adams, F. \& Fatuzzo, M. 1996, ApJ, 464, 256

André, Ph., Belloche, A., Motte, F., \& Peretto, N., 2007, A\&\&A, 472, 519

André, Ph., Basu, S., \& Inutsuka, S-I., 2009, in Structure Formation in Astrophysics, G. Chabrier Ed., Cambridge University Press

André, P., Men'shchikov, A., \& Bontemps, S. et al. AESA, in press, arXiv1005.2618

Bastian, N., Covey, K., \& Meyer, M., 2010, ARA\&A, 48

Basu, S. \& Jones, C., 2004, MNRAS, 347, L47

Bate, M., Bonnell, I., \& Bromm, V., 2003, MNRAS, 339, 577

Bate, M., 2009, MNRAS, 392, 1363

Bate, M. \& Bonnell, I., 2005, MNRAS, 356, 1201

Bonnell I., Bate M., Clarke C., \& Pringle J., 2001, MNRAS 323, 785

Chabrier, G., 2003, PASP, 115, 763

Chabrier, G. \& Hennebelle, P., 2010, ApJL in press, arXiv1011.1185

Clark, P., Klessen, R., \& Bonell, I., 2007, MNRAS 379, 57

Elmegreen B.G., 1997, ApJ 486, 944

Elmegreen, B., Klessen, R., \& Wilson, C., 2008, ApJ, 681, 365

Federrath, C., Roman-Duval, J., Klessen, R., Schmidt, W., \& Mac Low, M.-M., 2010, A\&A, 512,81

Heithausen, A., Bensch, F., Stutzki, J., Falgarone, F., \& Panis, J.-F., 1998, A\&AA, 331, L65

Hennebelle, P. \& Teyssier, R., 2008, A\&GA, 477, 25

Hennebelle, P., Banerjee, R., Vázquez-Semadeni, E., Klessen, R. \& Audit, E., 2008, A\&A, 446, 43

Hennebelle, P. \& Chabrier, G., 2008, ApJ, 684, 395 (HC08)

Hennebelle, P. \& Chabrier, G., 2009, ApJ, 702, 1428 (HC09)

Jappsen, A., Klessen, R., Larson, R., Li, Y., \& Mac Low,M.-M., 2005, A\&A, 435, 611

Kritsuk, A. G., Norman, M. L., Padoan, P., \& Wagner, R. 2007, ApJ, 665, 416

Kroupa P., 2002, Sci. 295, 82

Kunz M. \& Mouschovias T., 2009, MNRAS 399L, 94

Larson R., 1973, MNRAS, 161, 133

Larson R., 1981, MNRAS, 194, 809

Machida, M., Matsumoto, T., Hanawa, T., \& Tomisaka, K., 2005, MNRAS, 362, 382

McKee C.F. \& Ostriker J.P., 2007, ApJ 218, 448

McKee C.F. \& Tan T., 2003, ApJ 585, 850

MacLow, M.-M. \& Klessen, R., 2004, Rev. Mod. Phys., 76, 125

Myers P.C., 2009, ApJ, 706, 1341

Offner, S., Klein, R., McKee, C., \& Krumholz, M., 2009, ApJ, 703, 131

Padoan, P., Nordlund, A., \& Jones, B., 1997, MNRAS, 288, 145

Padoan, P. \& Nordlund, 1999, ApJ, 526, 279

Padoan, P. \& Nordlund, 2002, ApJ, 576, 870

Press, W. \& Schechter, P., 1974, ApJ, 187, 425

Price, N. \& Podsiadlowski, P., 1995, MNRAS, 275, 1041

Salpeter, E., 1955, ApJ, 121, 161

Scalo J., 1986, FCPh, 11, 1

Silk, J., 1995, ApJ, 438, L41

Shu F., 1977, ApJ, 214, 488

Schmidt, W., Federrath, C., Hupp, M., Kern, S., \& Niemeyer, J., 2009, A\&A, 494, 127 
Schmidt, W., Kern, S., Federrath, C., \& Klessen, R., 2010, A\&A, 516, 25

Smith, R., Clark, P., \& Bonnell, I., 2008, MNRAS, 391, 1091

Troland T. \& Heiles C., 1986, ApJ, 301, 339

Vázquez-Semadeni, E., 1994, ApJ, 423, 681

Zinnecker H., 1982, in Glassgold A. E. et al., eds, Symposium on the Orion Nebula to Honour

Henry Draper. New York Academy of Sciences, New York, p. 226

Zinnecker H., 1984, MNRAS, 210, 43

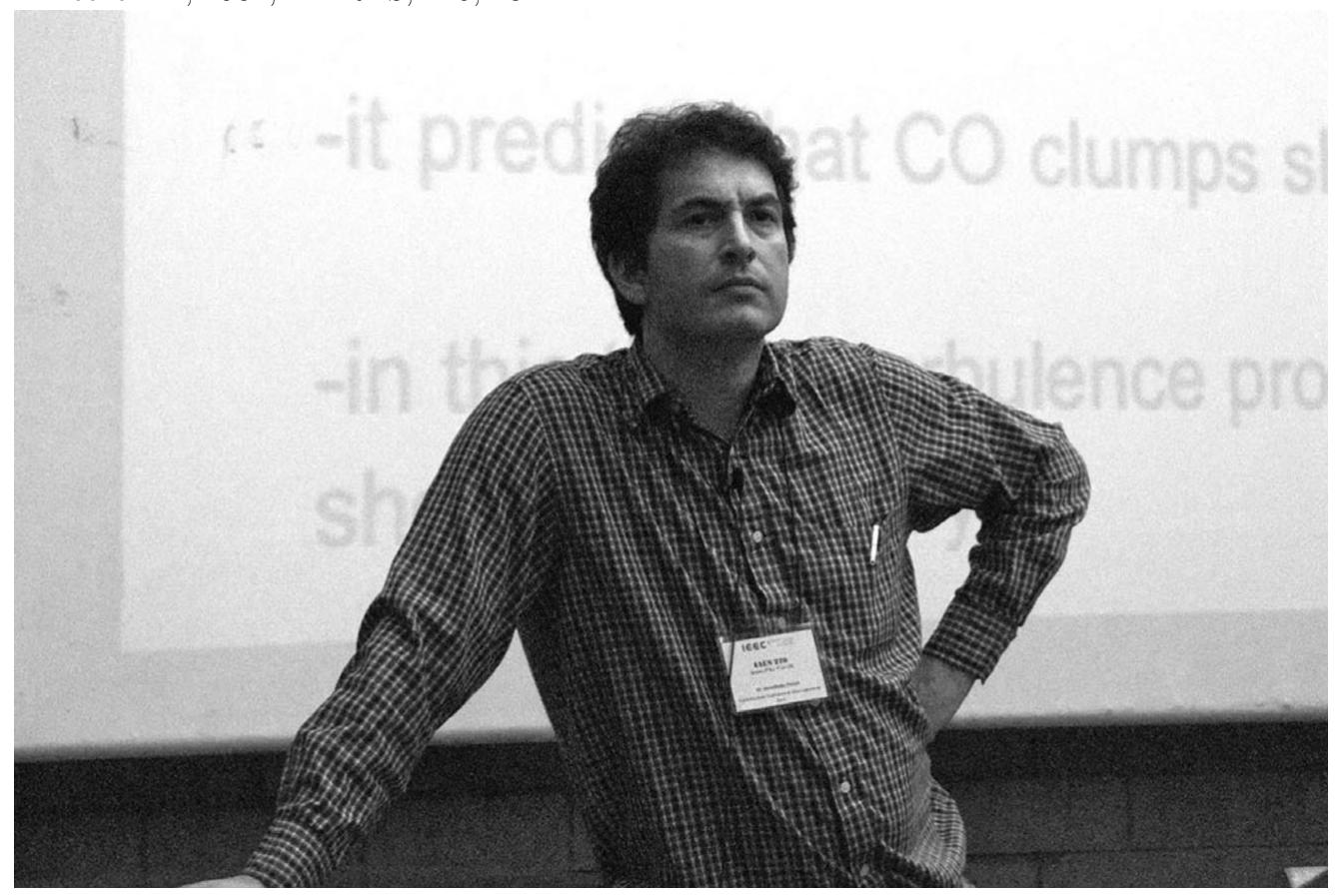

Patrick Hennebelle 